\title{
Discussion to 66 Giant Aneurysms of the Internal Carotid Artery
}

\author{
Shiro Waga, Kinya Ohtsubo, \\ Masayuki Matsuda and Hajime Handa \\ Department of Neurosurgery, Kyoto University \\ Medical School
}

Intracranial aneurysms of a diameter of $2.5 \mathrm{~cm}$ or greater are defined as giant. We have experienced 7 such giant aneurysms originated from the internal carotid artery. Six were female and I was male. Five were over the age of 50 and 4 were hypertensive. Three were located in the infraclinoid intracavernous portion and other 3 in the supraclinoid suprasellar portion, all 6 showing cranial nerve involvement without subarachnoid hemorrhage. There were 2 internal carotidposterior communicating aneurysms, both of which developed subarachnoid hemorrhage.

Two IC-PC giant aneurysms were explored and found to be greater than those observed on the angiograms. In the 2 other aneurysms, the size of calcification of the aneurysms on the plain skull films was far greater than that of the aneurysms on the angiograms.

In one, bilateral giant aneurysms of the internal carotid artery, suprasellar on the left and infracinoid on the right, were found.

The results of treatment were not good. Only one who had carotid ligation in the neck for the infraclinoid giant aneurysm showed improvement. Intracranial exploration and trapping for 2 IC-PC giant aneurysms resulted in postoperative death in one and in unchanged neurological status in the other. Other 4 had no surgical treatment. And one died within a year and the other 3 were still alive, showing unchanged neurological deficits.

Bull (1969) expressed his opinion that the most disappointing aspect of massive aneurysms was the treatment. The giant aneurysm which act as space-occupying lesion should ideally be excised. At the present level of surgical technique, however, the excision of giant aneurysm is one of the most difficult tasks or even impossible, because of its large size, its extremely broad neck and its complicated relationship to the parent vessel and the branching vessels or to the neighboring nervous strctures. 